\title{
Measurements of Jet and Multijet Cross Sections with the CDF Detector
}

\author{
Matthias Tönnesmann ${ }^{1,2}$ (Representing the CDF Collaboration) \\ 1 Max-Planck-Institut für Physik, Föhringer Ring 6, 80805 München, Germany \\ ' 2 Department of Physics and Astronomy, Michigan State University, East Lansing, MI 48824, U.S.A. \\ Received: date / Revised version: date
}

\begin{abstract}
Recent measurements of jet and multijet production cross sections from $p \bar{p}$ collisions recorded with the Collider Detector at Fermilab (CDF) are summarized. First Run II results of the inclusive one jet cross section at $\sqrt{s}=1.96 \mathrm{TeV}$ as well as prospects for future extensions of this measurement are presented. We also studied the properties of three-jet events in Run Ib data at $\sqrt{s}=1.8 \mathrm{TeV}$. All results are compared to predictions of Quantum Chromodynamics at next-to-leading order perturbation theory.
\end{abstract}

PACS. 12.38.Qk QCD, Experimental tests - 13.87.Ce Jets in large-Q² scattering, Production

\section{Inclusive one jet cross section}

One of the most important goals of QCD measurements at hadron colliders is the extraction of the input parameters of the theory, the strong coupling constant $\alpha_{S}$ and the parton distribution functions (p.d.f.). The production of hadronic jets at the Tevatron also probes the highest momentum transfer region currently accessible and thus is potentially sensitive to a wide variety of new physics.

CDF Run I data 1] exhibited an excess in the inclusive jet cross section at high $E_{T}$ when compared to QCD predictions at next-to-leading order (NLO) using thencurrent parton distribution functions. This excess can be explained by an underestimated gluon content of the proton at high momentum fraction $x$. Indeed, the gluon distribution is not well constrained at high $x$ and has increased in recent p.d.f. fits 2, leading to better agreement with both the CDF and DØ inclusive jet cross section measurements.

In Run II the measurement of jet production and the sensitivity to new physics will profit from the large integrated luminosity and the higher cross section, which is associated with the increase in the center-of-mass energy from $1.8 \mathrm{TeV}$ to $1.96 \mathrm{TeV}$.

\subsection{Status of Run II measurement}

The results presented here are based on data recorded from February 2002 through January 2003 corresponding to an integrated luminosity of $85 \mathrm{pb}^{-1}$. We have utilized the same techniques used in the previous CDF Run I inclusive jet analysis [1. In particular, we apply the Run I cone algorithm (JETCLU [3, $\left.R_{\text {cone }}=0.7\right)$ to reconstruct jets in the central pseudorapidity region $(0.1<|\eta|<0.7)$. Events were collected using 4 different $E_{T}$ trigger thresholds with appropriate prescale factors. To reduce background from cosmic rays, accelerator losses, and detector noise, cuts on the missing $E_{T}$ significance, $\widetilde{E_{T}}=\mathscr{E}_{T} / \sqrt{\sum E_{T}}$, are applied. A good energy measurement of jets is ensured by requiring the event vertex to be within $60 \mathrm{~cm}$ of the center of the detector along the beam direction.

The measured jet energies are corrected for experimental effects stemming from non-uniformities of the calorimeter response, multiple interactions, calorimeter non-linearity, and energy due to the underlying event. Since we currently rely on the absolute energy corrections determined in Run I, the jet energy scale has been set to that of Run I, thereby introducing a systematic uncertainty of $5 \%$, which is the dominant experimental systematic error. Further understanding of the energy scale will reduce this uncertainty.

The unsmeared jet cross section is shown in Fig. 1 (left). It is compared to a QCD prediction at NLO, which reproduces the distribution of the data well over 8 orders of magnitude. The theoretical prediction was calculated using the EKS program [4] and the CTEQ 6.1 p.d.f. set [5]. The renormalization and factorization scales were set to $E_{T} / 2$. The CTEQ 6.1 set of p.d.f. has available complete error information, which makes it possible to calculate the p.d.f. errors on the Run II jet cross section predictions, indicated as the curves in Fig. 10 The dominant p.d.f. uncertainty comes from the gluon density at high $x$, which is the least well constrained parameter of the CTEQ 6.1 p.d.f. set. The full Run II dataset will help to reduce this uncertainty (see Sect. 1.2).

The effect of the higher jet cross section in Run II is especially prominent at the high $E_{T}$ frontier, where two new bins were added. With a data sample similar in size 

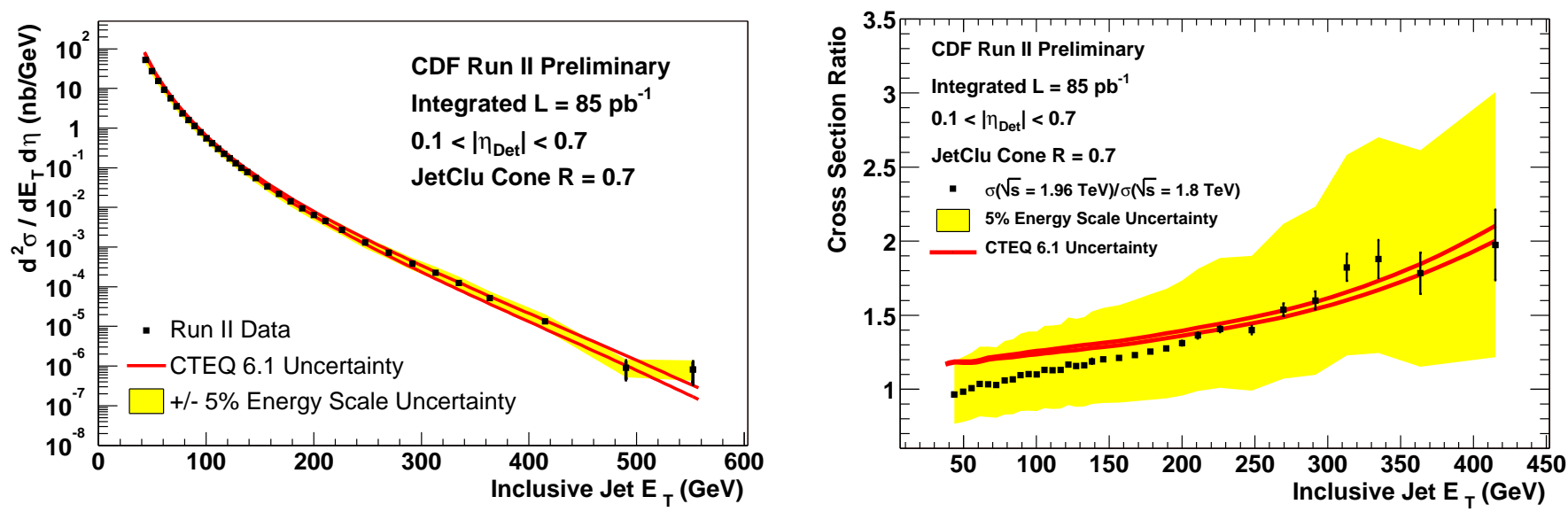

Fig. 1. (Left) Comparison of the measured inclusive jet cross section from Run II data (points) to QCD predictions at NLO using CTEQ 6.1 p.d.f. (curves). (Right) Run II/Run I cross section ratio compared to the QCD prediction at NLO using CTEQ 6.1 p.d.f.

to that obtained in Run Ib, we are thus already able to extend the $E_{T}$ range covered by the Run I measurements by almost $150 \mathrm{GeV}$. The Run II/Run I cross section ratio, together with a QCD prediction at NLO, is shown in Fig. 1 (right). The ratio is seen to be lower than expected at low $E_{T}$, but we find good agreement within the experimental and theoretical uncertainties.

\subsection{Prospects for future extensions}

A powerful way to understand the nature of a potential excess in the jet cross section at high $E_{T}$ is the extension of the analysis described above into the forward region of the detector. The new CDF endplug calorimeters, which cover the pseudorapidity range $1.1<|\eta|<3.6$, will permit such a measurement. Forward jet measurements are not expected to have any contribution from new physics because the maximum reachable $E_{T}$ is limited to, e.g., about $200 \mathrm{GeV}$ for $2.1<|\eta|<2.8$. On the other hand the sensitivity to the gluon distribution in the proton is similar to that of central jet measurements. The gluon distribution at high $x$ can thus be further constrained, which will in turn increase the sensitivity to new physics in the high $E_{T}$ (high mass) region of the central one jet (di-jet) cross section.

Another improvement in jet measurements can be attained by the use of other jet reconstruction algorithms. CDF has so far relied on its cone algorithm JETCLU 3 to search for jets, define jet observables and measure jet cross sections. During the past few years different theoretical problems of cone algorithms were pointed out [6], namely the infrared and collinear sensitivity of the observables, e.g. cross sections, and the difficulty to match the experimental algorithms with those employed in theoretical calculations. Besides improved cone algorithms, the longitudinally invariant $k_{T}$ clustering algorithm [7] will be an important tool because of its built-in infrared and collinear insensitivity and its direct applicability at the parton and at the detector level.
Fig. 2 shows the ratio of raw (uncorrected) inclusive one jet cross sections using the $k_{T}$ clustering algorithm with the angular jet separation parameter $D$ set to 0.7 and 1.0 and the JeTCLU algorithm $\left(R_{\text {cone }}=0.7\right)$. Events were selected as described in Sect. 1.1 For $D=0.7$ the uncorrected $k_{T}$ cross section is about $5 \%$ lower than the JETCLU cross section, while $D=1.0$ produces bigger jets with larger $E_{T}$, which directly translates into a $20 \%$ increase in the cross section. Furthermore we observe an increase of the ratio at low $E_{T}$, which is qualitatively similar to the low $E_{T}$ behavior of the ratio of fully corrected and unsmeared cross sections measured by D $\varnothing$ using $k_{T}$ jets $(D=1.0)$ in the Run I data sample $[8$. It is important to note, however, that different jet algorithms may have different energy corrections. In particular, the correction for the underlying event will be larger for $k_{T}$ jets with $D=1.0$ than for $D=0.7$. Quantitative comparisons should therefore be carried out only after correcting the jet energies and unsmearing the cross section distribution.

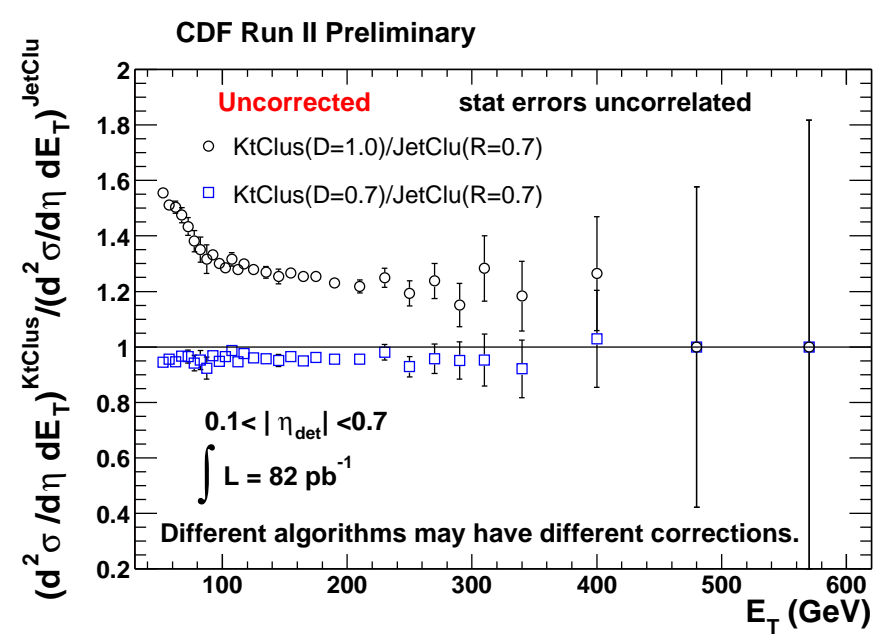

Fig. 2. Ratio of raw jet cross sections using the $k_{T}$ clustering algorithm $(D=0.7,1.0)$ and the JETCLU cone algorithm $\left(R_{\text {cone }}=0.7\right)$ from Run II data. 

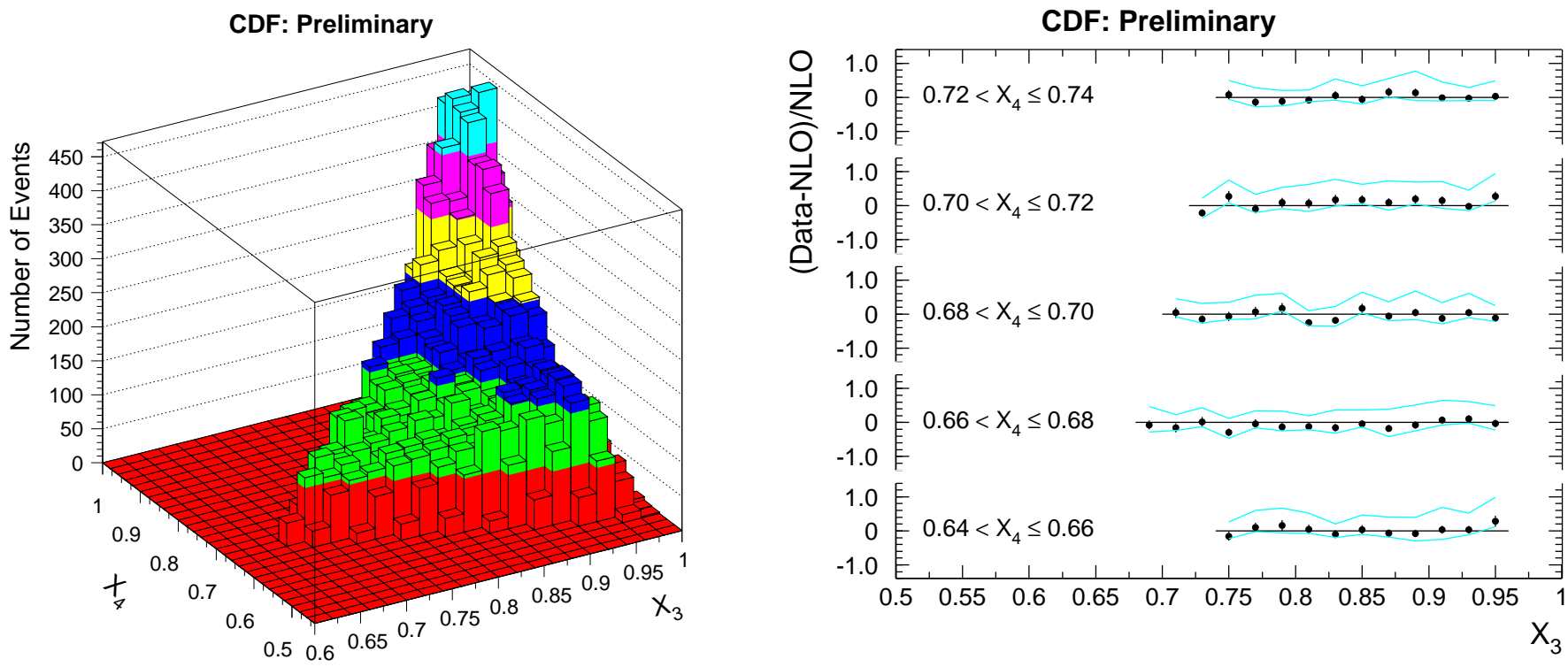

Fig. 3. (Left) Measured distribution of the 3-jet events in the $X_{3}-X_{4}$ plane from Run Ib data. (Right) Ratio (Data-NLO)/NLO for the differential cross section as a function of $X_{3}$ in the region $0.64<X_{4} \leq 0.74$. The band between the two curves represents the experimental systematic uncertainties.

The long term goal is to measure the inclusive jet cross section using the improved Run II cone algorithm and the $k_{T}$ clustering algorithm, and to extend the measurements to the forward region.

\section{Three-jet cross section}

With the availablitity of QCD predictions at NLO for the production of 3-jet events at hadron colliders [9] new possibilities for precision tests of QCD have opened up, among them the measurement of $\alpha_{S}$ from the ratio of 3-jet and 2 -jet production rates or from event shapes. A different approach is the analysis of the topology of 3-jet final states using Dalitz variables, which will be presented in the following.

We analyzed $86 \mathrm{pb}^{-1}$ of Run Ib data. Jets are reconstructed using the JETCLU algorithm with $R_{\text {cone }}=0.7$. 3 -jet events are selected by requiring at least 3 jets with $E_{T} \geq 20 \mathrm{GeV}$ and $|\eta|<2.0, \sum E_{T}(3$ jets $)>320 \mathrm{GeV}$, and a separation of $\Delta R>1.0$ in the eta-phi plane between the jets. The events are boosted into the 3-jet rest frame, and the 3 leading jets are numbered such that $E_{3}>E_{4}>$ $E_{5}$. The 3 -jet mass $m_{3 \text {-jet }}$ is calculated, together with the Dalitz variables $X_{i}=2 E_{i} / m_{3-\text { jet }}, X_{3}+X_{4}+X_{5}=2$.

Fig. 3 (left) shows the measured distribution of the 3 -jet events in the $X_{3}-X_{4}$ plane. The topologies are dominated by configurations containing a soft third jet.

The differential cross section as a function of $X_{3}$, measured in different bins of $X_{4}$, was compared to QCD calculations at NLO using the CTEQ 4M p.d.f. Fig. B (right) shows the relative difference between data and theory in the region $0.64<X_{4} \leq 0.74$. Reasonable agreement was observed in the whole $X_{3}-X_{4}$ plane. The experimental systematic uncertainties are dominated by the jet energy scale.
The total 3-jet production cross section, integrated over the $X_{3}-X_{4}$ plane with $X_{3}<0.98$, yields $\sigma^{3 \text {-jet }}=$ $456 \pm 2$ (stat. $)_{-68}^{+202}$ (syst.) $\mathrm{pb}^{-1}$, consistent with the NLO prediction $\sigma_{\mathrm{NLO}}^{3-\text { jet }}=482 \pm 2$ (stat. $)_{-72}^{+31}$ (theo.) $\mathrm{pb}^{-1}$. The theoretical uncertainty is due to the arbitrary choice of the renormalization and factorization scales, and was calculated by varying the scales by factors of 0.5 and 2 .

The NLO predictions have also been calculated using different members of the CTEQ 4A p.d.f. family [10], which differ from CTEQ 4M in the value of $\alpha_{S}$. However, an extraction of $\alpha_{S}$ from a $\chi^{2}$ analysis is not possible due to lack of sensitivity to $\alpha_{S}$ within the large uncertainties.

\section{Acknoledgments}

I would like to thank the European Commission and the European Physical Society for financial support.

\section{References}

1. T. Affolder et al. (CDF Coll.), Phys. Rev. D 64, (2001) 032001

2. J. Pumplin et al., J. High Energy Phys. 07, (2002) 012

3. F. Abe et al. (CDF Coll.), Phys. Rev. D 45, (1992) 1448

4. S.D. Ellis, Z. Kunszt, D.E. Soper, Phys. Rev. Lett. 62, (1989) 726; 64, (1990) 2121; Phys. Rev. D 40, (1989) 2188; Z. Kunszt, D.E. Soper, Phys. Rev. D 46, (1992) 192

5. D. Stump et al., J. High Energy Phys. 10, (2003) 046

6. G.C. Blazey et al., FERMILAB-CONF-00-092-E

7. S.D. Ellis, D.E. Soper, Phys. Rev. D 48, (1993) 3160

8. V.M. Abazov et al. (DØ Coll.), Phys. Lett. B 525, (2002) 211

9. W.B. Kilgore, W.T. Giele, hep-ph/0009193

10. H.L. Lai et al., Phys. Rev. D 55, (1997) 1280 